\title{
Structurer la demande de formation des personnels dans les établissements d'enseignement secondaire
}

Eric Auziol, Richard Etienne et Nadine Milhaud

\section{OpenEdition}

Journals

Édition électronique

URL : http://journals.openedition.org/trema/2230

DOI : 10.4000/trema.2230

ISSN : 2107-0997

Éditeur

Faculté d'Éducation de l'université de Montpellier

\section{Édition imprimée}

Date de publication : 1 décembre 1994

Pagination : 31-43

ISSN : 1167-315X

\section{Référence électronique}

Eric Auziol, Richard Etienne et Nadine Milhaud, «Structurer la demande de formation des personnels dans les établissements d'enseignement secondaire », Tréma [En ligne], 6 | 1994, mis en ligne le 01 décembre 1994, consulté le 19 avril 2019. URL : http://journals.openedition.org/trema/2230 ; DOI : 10.4000/trema.2230

Ce document a été généré automatiquement le 19 avril 2019.

Trema 


\title{
Structurer la demande de formation des personnels dans les établissements d'enseignement secondaire
}

\author{
Eric Auziol, Richard Etienne et Nadine Milhaud
}

1 Le système éducatif se prépare à franchir une nouvelle étape : $80 \%$ d'une classe d'âge au niveau du baccalauréat. Ce saut quantitatif induit des objectifs nouveaux et une rénovation de l'organisation scolaire. Quels leviers permettront d'opérer les transformations indispensables à cette évolution? La formation continue peut être l'un d'eux, à la condition qu'elle réponde réellement aux demandes suscitées par cette rénovation. Elle ne peut donc plus obéir à une seule logique de l'offre, liée aux points de vue et aux compétences des seuls formateurs. Elle doit devenir une formation qui répond à des besoins, à des demandes, voire même à une commande formulés par les acteurs du changement, ce qui la situe à la rencontre d'une logique de la demande et d'une logique de l'offre.

\section{De l'offre de formation à la demande : quelle signification donner à une telle transformation?}

2 Cette logique de la demande s'inscrit dans l'évolution des mentalités. Depuis que la notion de formation (continue ou initiale) est entrée dans nos sociétés, les comportements évoluent. On veut être acteur de sa propre formation, et ce d'autant plus que certaines actions "subies" se sont révélées inadéquates aux besoins. Nous remarquons également une volonté politique de responsabiliser les institutions et les acteurs avec le souci d'une efficacité plus grande. L'obligation faite à tous les niveaux d'élaborer des projets - projets d'actions académiques, projet d'établissement, projet de formation, projet personnel de l'élève (ETIENNE R. et al., 1992), etc.- est, sans doute, un moyen susceptible d'amener à la réflexion, à la compréhension, à la prise d'initiatives et à l'adhésion par l'engagement. La réalisation de ces projets fait apparaître des besoins en formation susceptibles de 
déboucher sur des demandes ou des commandes de formation. Mais quels sont les besoins en formation? Quels sont les lieux de leur expression et les partenaires qui participent à leur analyse? La typologie présentée ci-dessous s'appuie sur un modèle fonctionnel et explicatif, elle ne met donc pas en évidence les multiples interactions qui règlent le mouvement du système.

\section{Une logique politique}

Les besoins nés d'une commande descendante sont communs à tous les établissements. L'institution centrale définit des orientations générales, détermine des objectifs et, parfois même, met en place des dispositifs (par exemple, dispositif d'évaluation en seconde destiné à repérer le niveau des élèves et à faciliter l'apport de réponses aux difficultés constatées).

\section{Figure 1 : Les besoins induits par la commande de la société et de l'administration centrale}

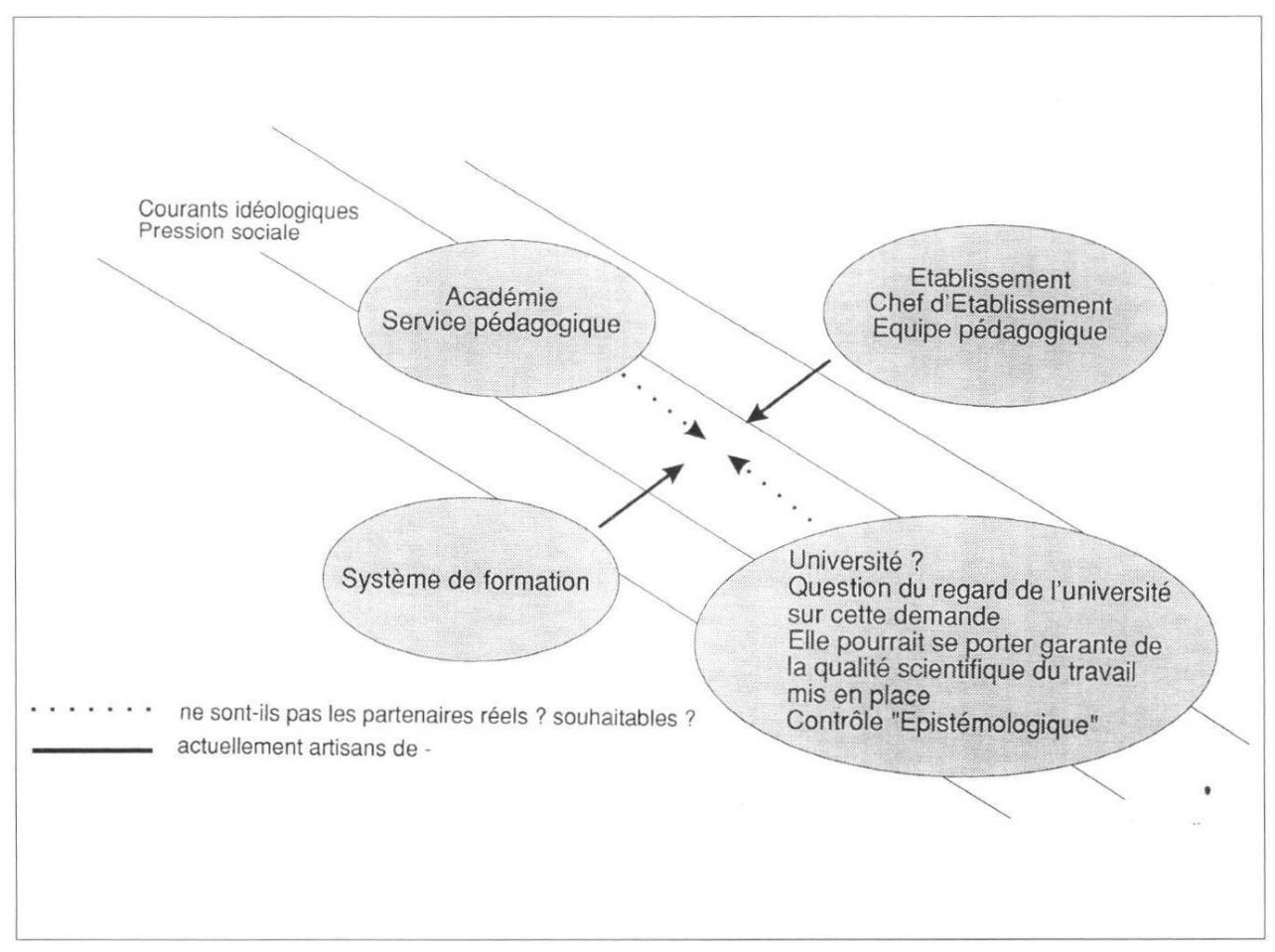

4 Un fonctionnement idéal voudrait que les services administratifs et académiques du rectorat et le système de formation, sous la vigilance de l'Université déterminent, en relation avec les établissements scolaires (chefs d'établissement, équipes pédagogiques), un certain nombre de besoins propres à l'académie, en déduisent une orientation et des objectifs qui permettent d'élaborer une demande, voire de passer une commande de formation. La réalité est toute différente et c'est bien souvent de la seule confrontation du système de formation et des services pédagogiques du rectorat que naît la demande.

\section{Une logique environnementale}

5 Les besoins liés à l'établissement et à son environnement (nous pourrions parler des besoins liés à l'emploi et au bassin d'emploi) sont spécifiques. Chaque établissement 
présente des caractères propres; ils sont liés à la population qu'il accueille, à l'environnement, à son histoire... Pour atteindre un objectif donné, l'établissement recense les besoins en formation liés à ces caractères. Puis, les demandes de formation correspondantes sont communiquées à la Mission Académique (MAFPEN) dans le cadre du Plan Académique de Formation (PAF).

Figure 2 : Les besoins induits par l'établissement et son environnement

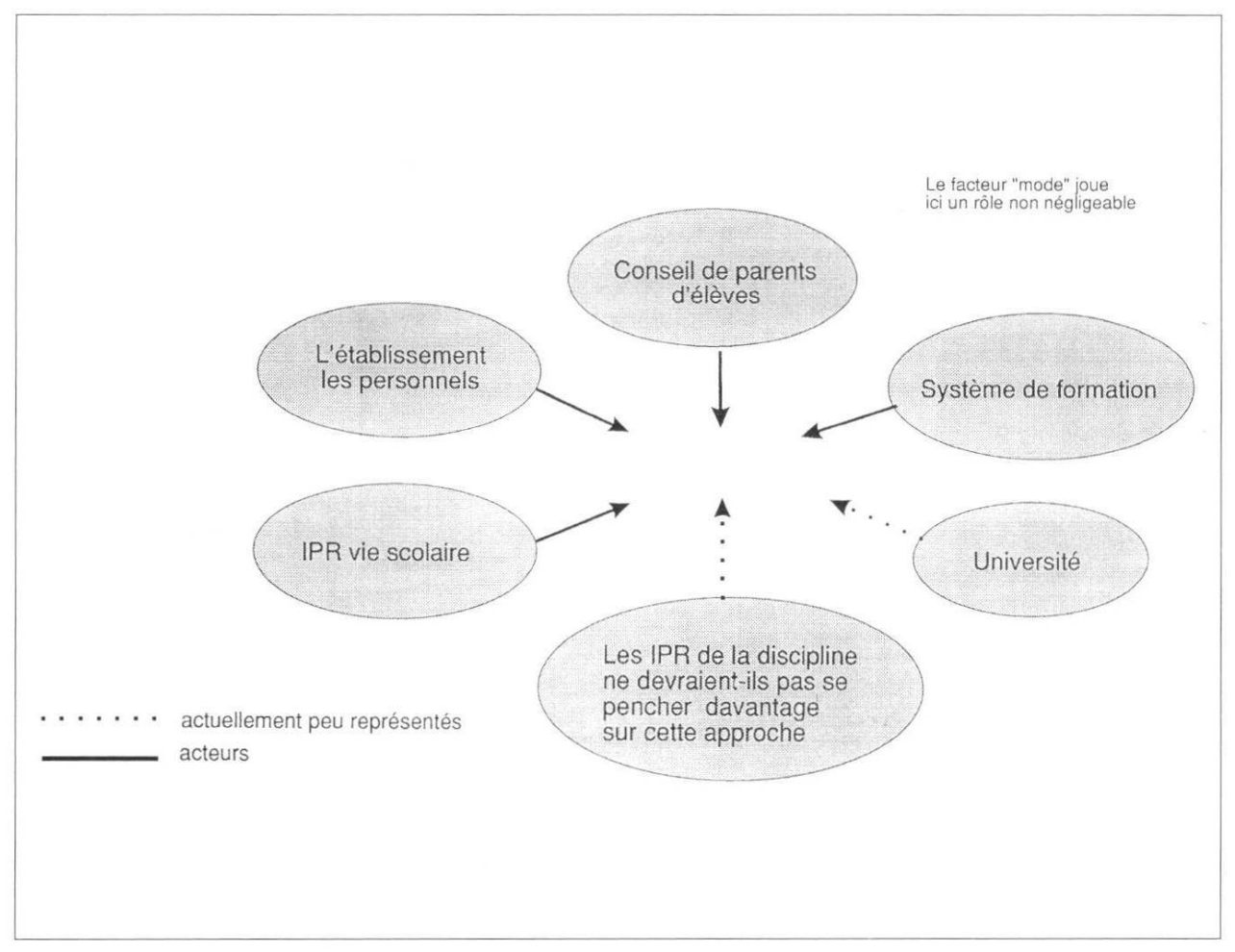

6 L'analyse des besoins et le passage à une demande devraient être menés conjointement par l'établissement, l'Inspecteur Pédagogique Régional-Inspecteur d'Académie (IPR-IA) Vie Scolaire, le conseil de parents d'élèves, les IPR-IA de disciplines, les Universités et le système de formation. Dans la réalité (figure 2), seuls les trois premiers partenaires participent effectivement à cette étude.

\section{Une logique personnelle}

7 Les besoins personnels liés à la profession sont individuels ou communs à de petits groupes de personnes. Dans ce cas (figure 3), pour les enseignants, l'analyse des besoins est essentiellement faite par les professeurs eux-mêmes, par l'IPR-IA de la discipline et, parfois, par le système de formation. L'Université est absente. Pourtant, en ce qui concerne la didactique et les contenus académiques, son rôle dans la transformation du besoin en demande pourrait être primordial. 


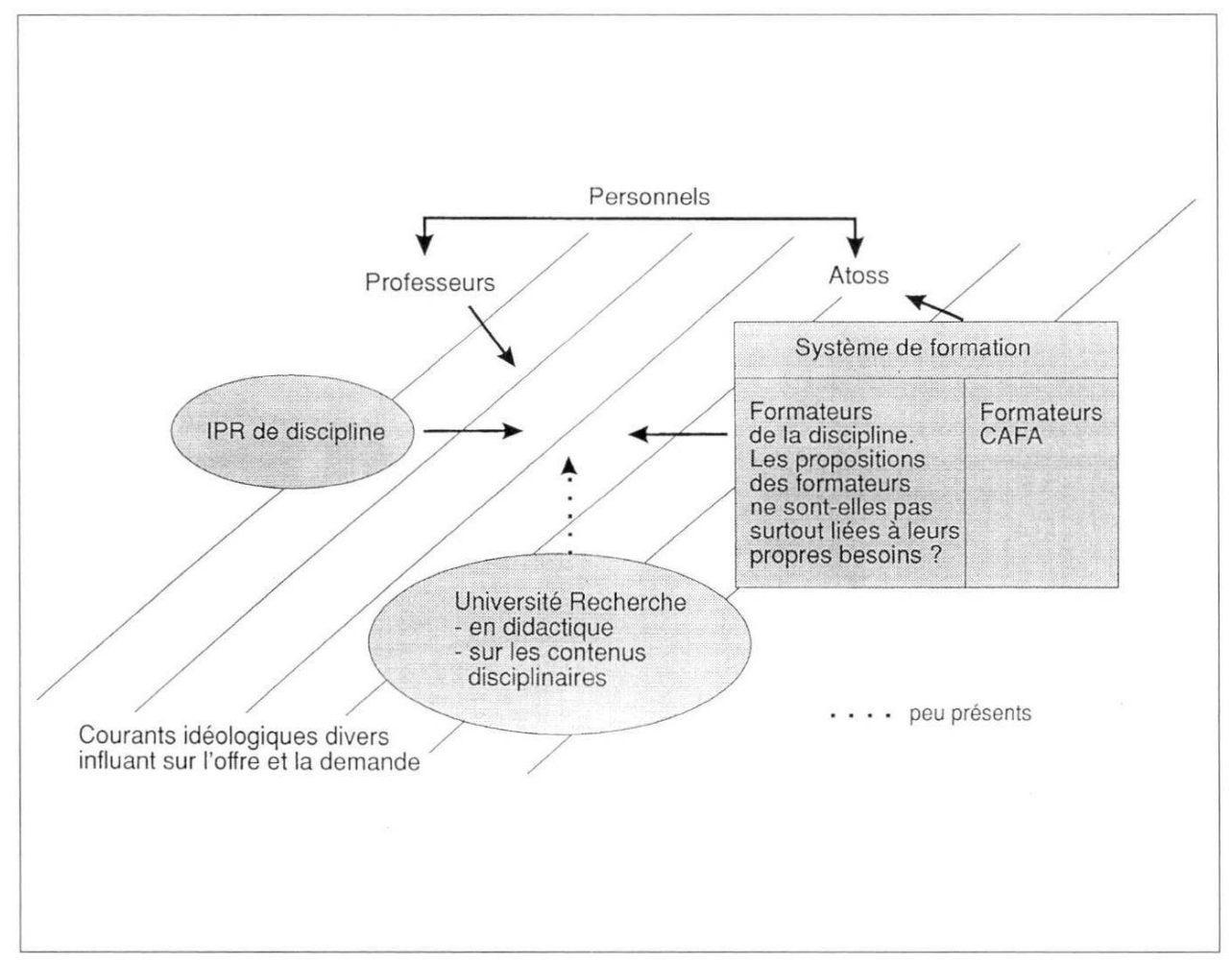

Bien que tous les acteurs du système éducatif soient convaincus de l'importance de la formation et de la nécessité impérative pour l'évolution du système de la mise en place d'une vraie politique facilitant l'émergence de la demande, ils ne participent qu'irrégulièrement aux instances mises en place dans cette intention.

\section{La faiblesse de la demande: quels freins au changement?}

9 Pour expliquer cette faiblesse déjà constatée dans les années 1980 (tant qualitative que quantitative), nous essaierons d'en analyser les causes structurelles, historiques et culturelles, en abordant successivement la place de la formation des personnels dans l'Education Nationale, la clôture de la classe et de l'établissement scolaire avant de terminer par le déficit de communication sur la formation.

\section{La formation des personnels, "passager clandestin"}

La formation des enseignants et de ceux qui contribuent au service d'éducation précédait traditionnellement leur entrée en fonction. L'introduction récente (rapport de PERETTI. 1982) de leur formation continue découle des lois Chaban (1974), mais s'est faite dans le respect d'anciens équilibres. En effet, il ne fallait ni toucher aux congés des enseignants, ni diminuer de façon trop visible les horaires d'enseignement reçus par les élèves. $\mathrm{Ce}$ choix a entraîné trois conséquences :

1. la formation continue n'est pas une obligation. Un enseignant peut passer les 37 ans $1 / 2$ de sa carrière sans recevoir de formation, quels que soient les changements profonds qui 
s'opèrent dans les programmes et méthodes d'enseignement. Mais l'obligation de formation n'entraînerait-elle pas un remodelage sérieux des structures?

2. les durées moyennes des actions de formation n'excèdent que rarement trois jours (il faut alors procéder à des remplacements - cas de l'informatique pédagogique - ou à des tours de passe-passe qui permettent de ne pas inscrire, provisoirement, une discipline d'enseignement dans l'emploi du temps des élèves - variante rencontrée lors de la formation-reconversion des professeurs de technologie),

3. un établissement qui choisit une ou des actions collectives doit avoir la force de se mettre "en panne d'enseignement" pour la durée de cette formation.

11 Comme la décentralisation a renforcé le droit de regard des parents, les stages suivis par les enseignants peuvent susciter des conflits avec l'administration. L'actuelle rigidité des emplois du temps, l'absence de travail en équipe et, il faut l'avouer, un certain manque d'imagination exercent une pression qui tend au conformisme. "Les enseignants n'ont pas à se former, puisqu'ils ont d'abord à former les élèves qui leur sont confiés", ainsi pourrait être caricaturée cette attitude. Elle pèse d'autant plus que le nombre de personnes devant partir en formation est élevé et que l'ensemble des partenaires ne se donne ni moment ni lieu pour poser le problème de la formation continue des diverses catégories de personnels.

C'est qu'il y a une assimilation du départ en formation à "l'absentéisme". Aussi, pour diminuer le taux de cours non-assurés, est-il tentant de fermer le seul robinet que l'on puisse maitriser: celui des stages. Pourtant, la formation renforce la motivation des enseignants et induit une diminution des congés de courte durée (étude réalisée sur plusieurs collèges en rénovation des Pyrénées-Orientales). Ces faits confirment la rentabilité de l'investissement-formation (MEIGNANT A., 1986). Il n'en reste pas moins vrai que, même si le taux d'absentéisme de ce personnel est faible, toute mission confiée à un enseignant se traduit, la plupart du temps, par un déficit de cours durement ressenti par les parents d'élèves.

De plus, la gestion des formations est faiblement concertée au sein des rectorats: les Missions Académiques à la Formation des Personnels de l'Education Nationale (MAFPEN) restent des structures très légères. Elles n'ont pas la responsabilité d'évaluer les effets de la formation sur les apprentissages des élèves. De nombreux stages portent sur des thèmes transversaux, ce qui laisse de côté l'identité disciplinaire des enseignants

Enfin, une pratique souhaitable et souhaitée par de nombreux établissements, la libre détermination de leur plan de formation, aboutit à un effet inattendu : si un enseignant est "trop souvent parti en formation", il n'aura que la liberté de renoncer, de lui-même ou sur injonction du chef d'établissement ou encore de la rumeur, à un stage qu'il a pourtant demandé et qui a été programmé par la MAFPEN !

15 La formation continue a été introduite sans que l'ensemble du système soit repensé pour qu'elle y ait réellement sa place. Elle est tolérée, elle est, en quelque sorte, le passager clandestin que l'on ne peut exclure, mais qui ne doit pas gêner la progression du navire ni ne peut influer sur sa direction.

\section{La clôture de la classe et de l'établissement}

16 La classe demeure le lieu où se pratique l'acte éducatif; l'enseignant y déploie une activité très "artisanale" et les observations concordent (POSTIC M. et de KETELE J.-M., 1988) pour établir que la solitude de l'enseignant confine à l'isolement. Il se crée un 
ensemble de palliatifs, de conduites prédéterminées qui lui permettent de régler le cours de son enseignement. Cette pratique tient lieu de "formation spontanée" (AUZIOL E. et NEGRELL M., 1983), fait reculer la socialisation des enseignants et anéantit toute expression d'une demande perçue comme inutile. Il n'est donc pas surprenant de constater que la faiblesse du travail en équipe se traduit par une absence de réflexion collective sur la formation.

De leur origine religieuse, les établissements scolaires ont gardé le principe de la double clôture : isolement du monde extérieur, mais aussi cloisonnement intérieur, la tentation étant grande pour chacun de revendiquer "sa" salle de classe! C'est ainsi qu'aujourd'hui encore, les espaces de communication et de travail en équipe, se réduisent à la seule salle des professeurs dont la polyvalence aboutit à en faire un espace de convivialité plus qu'un lieu de travail. La diversité des statuts des enseignants et la multiplicité des disciplines scolaires renforcent les tendances à l'individualisme.

Quant au temps de l'univers scolaire, il est, lui aussi, pensé comme un ensemble de tranches d'enseignement, généralement horaires, qu'il convient de disposer au mieux pour que l'acte éducatif réussisse. Quelques rares innovations dans ce domaine (journée de prérentrée et conseils d'enseignement) n'ont pu donner leur plein effet. Les enjeux de ces journées (remise des emplois du temps individuels et choix des manuels de classe) interdisent d'y mener tout autre type d'activités.

Dans ces conditions, la faiblesse de la demande actuellement constatée se traduit sous des formes très contrastées : accès de fièvre précédant des périodes d'arrêt total instaurées par compensation. Quelques expériences réussies dans un passé récent incitent à penser qu'il y aurait intérêt à instituer une réflexion collective sur la formation, parmi les personnels d'abord, puis au sein d'instances dont la vocation est de prendre des décisions, comme le Conseil d'Administration. Le débat sur la formation ne peut s'envisager que dans le cadre d'une conception nouvelle de l'établissement scolaire, de son rôle dans le système éducatif et de sa communication avec l'extérieur (ETIENNE R. et AMIEL M., 1994). Les lois de décentralisation et les décrets qui les ont accompagnées ont posé quelques jalons vers une plus grande prise en compte de la société civile dans les décisions prises par le système éducatif. Le rôle du chef d'établissement y a gagné en importance quand il facilite, par son action et par son attitude, la transition vers un collège ou un lycée fonctionnant par projets individuels et collectifs.

\section{L'identité professionnelle des enseignants comme frein au développement de la demande de formation}

Depuis le Moyen-Age, l'enseignement est réservé à une corporation (universitas) composée de ceux qui savent, les maîtres (magistri, ceux qui occupent de grandes responsabilités, les magistères, par opposition aux petites, les ministères). Les valeurs républicaines et laïques, tout aussi absolutistes, sont venues déposer d'autres sédiments dans la représentation mentale du métier (OBIN J.-P., 1993). Toutefois, le professeur est d'abord celui qui sait et ce lieu commun ne facilite pas l'émergence de sa demande de formation, il la rend même illégitime, sauf si elle est neutralisée par la nature de son contenu (l'audiovisuel et l'informatique illustrent bien ce propos) ou par sa nouveauté (l'apparition de nouveaux savoirs). La hiérarchie des grades d'enseignants peut également jouer un rôle pervers dans l'élaboration de la demande de formation et le moindre succès (taux de demande moyen par enseignant) observé dans les lycées 
s'explique par une présence massive d'agrégés et de certifiés en fin de carrière. De plus, la forme même envisagée pour l'acte de formation traduit une nette recherche de la situation universitaire traditionnelle : ce qui prédomine, c'est le cours et la notion de recyclage sur un point très précis, notamment les derniers apports de la recherche. Ces observations, qui ne mettent pas en cause la nécessité de ce type de formation, expliquent pourquoi les demandes de formation se limitent à des démarches individuelles. Cette attitude se retrouve dans la distinction faite entre le droit à la formation que ne cessent de revendiquer les personnels et les besoins de formation que voudraient voir traités les hiérarchies pédagogique et administrative.

Or, tant que les personnels se comportent comme des "consommateurs de formation", ils sont en position d'attente, ils privilégient le catalogue ou la rumeur. S'ils entrent dans une démarche qui prend en considération l'ensemble du système, ils deviennent des investisseurs et forgent un projet individuel et/ou collectif. La formation peut être valorisée par le résultat qu'elle permet d'obtenir, mais il faut aussi la concevoir comme un moyen permettant d'atteindre un but qu'on s'est assigné.

$\mathrm{Au}$ niveau d'une académie, constater un manque de formation dans un domaine incite à offrir la possibilité de le combler : c'est l'époque du catalogue, l'enseignant s'inscrit à un stage, y va "pour voir" et revient dans son établissement où toute volonté de réinvestissement se heurte aux tendances lourdes du projet ou de la politique du collège ou du lycée. Pour l'institution, le coût du catalogue est modique, l'organisation qu'il implique reste très embryonnaire, mais son efficacité se révèle incertaine. La logique voudrait qu'on inscrive cette démarche dans une politique globale d'information qui n'est encore que balbutiante dans l'Education Nationale. C'est la rumeur, "le marché noir de l'information", (KAPFERER J.-N., 1987) qui véhicule le plus grand nombre de renseignements vrais ou faux sur la formation et les formateurs. Il peut en naître et il en naît souvent un décalage entre l'image perçue du système de formation et celle qu'il voudrait donner.

Suivant les intérêts et les enjeux du moment, les personnels se forgent des représentations qu'une politique systématique d'information aura bien du mal à faire évoluer. On ne lutte pas contre une rumeur par des catalogues ou des publications, mais par un maillage de personnes ressources présentes dans les établissements, elles-mêmes capables de saisir la complexité des données et d'en faire une présentation simple et convaincante. Concrètement, ces personnes peuvent s'investir dans une tâche indispensable mais encore peu pratiquée par et dans les établissements : l'évaluation des actions de formation et de leurs effets au niveau du collège, du lycée ou du bassin de formation. Cette évolution vers une plus grande prise en compte de la demande, pour logique qu'elle soit, s'est trouvée ralentie par les freins que nous avons évoqués, mais aussi et surtout par leur effet dans l'identité professionnelle des enseignants.

Le système de formation vise une transformation des représentations des personnels dans un triple domaine : la conception du métier, celle de la formation et celle du rôle de l'établissement scolaire (figure 4). Cette triple visée ne peut que s'accompagner de choix stratégiques et d'une volonté de discerner ce qui est possible de ce qui se révélerait illusoire. 


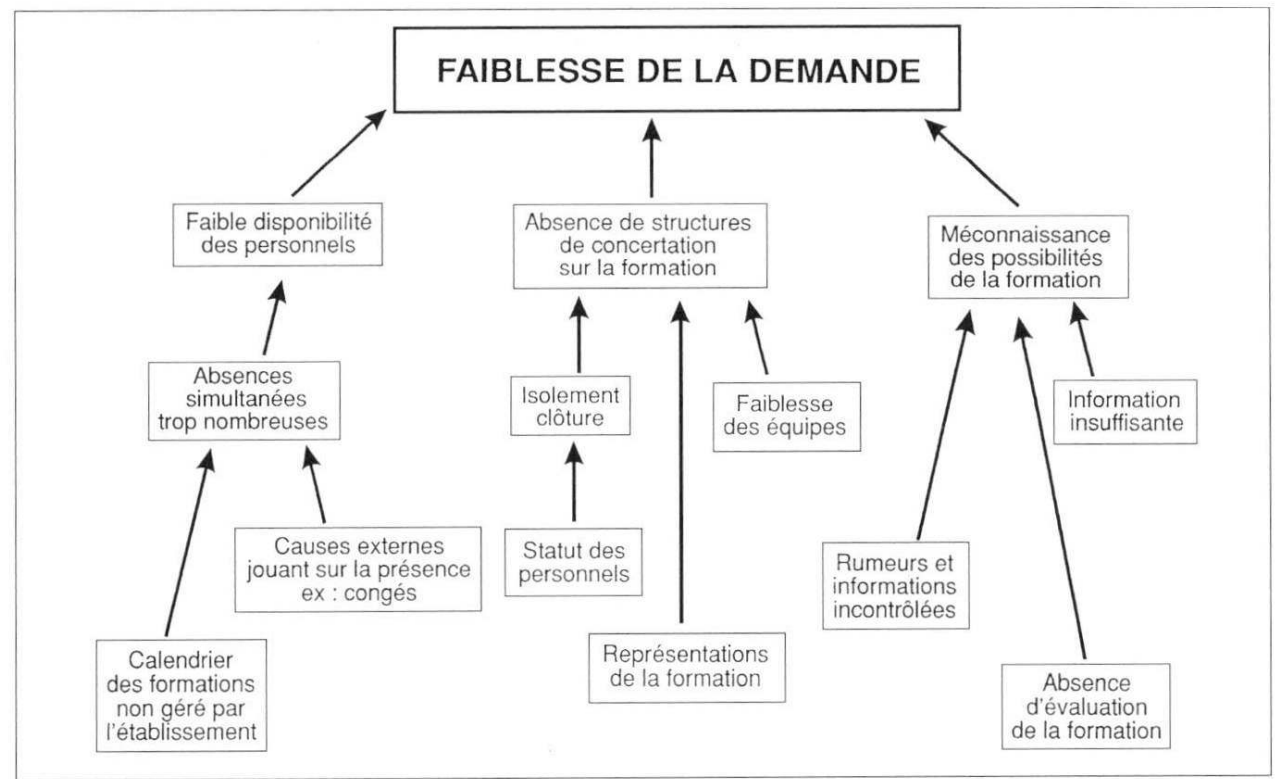

Se contenter d'attendre que les personnels demandent spontanément des actions de formation continue n'a jamais été la position de la hiérarchie. Toutefois, il convient de constater qu'elle n'a pas toujours su ou voulu mener une analyse stratégique pour expliquer et diminuer la faiblesse de cette demande. Dans l'organisation du système éducatif, de nombreuses contraintes doivent être réexaminées puis aménagées pour créer un environnement favorable à l'émergence de la demande et à son traitement; de même, au niveau de l'établissement, de chaque établissement, il convient de faire évoluer les structures pour créer un espace de concertation et de décisions négociées sur la formation. En définitive, toute politique vise à faciliter l'évolution des mentalités, donc l'enrichissement et la diversification des représentations. Or, elles sont actuellement encore trop marquées par des stéréotypes sur la formation, l'établissement et le métier d'enseignant.

\section{Quelles modifications pour développer l'expression de la demande?}

Est-il possible d'agir pour transformer cette situation que nous avons caractérisée par ses freins et ses logiques d'autolimitation de la demande de formation? Les travaux portant sur la communication, notamment autour de l'école de Palo Alto (WATZLAWICK P. et al., 1975), ont montré qu'il ne suffit pas de vouloir un changement pour que celui-ci commence à se réaliser. Bien au contraire, si les agents de changement se trouvent en position centrale (voire hiérarchique) dans l'institution, leur intervention produit souvent des effets opposés à ceux recherchés et concourt à reproduire les structures fermées, capitonnées dans leurs défenses rassurantes.

Pourtant, loin d'un pessimisme systématique, nous cherchons à repérer ce qu'il est possible de faire, quelles sont les modifications réelles susceptibles d'activer la demande de formation. Cela revient à identifier quelques points d'ancrage qu'une politique déterminée et tenace pourrait chercher à exploiter. 


\section{Décentraliser systématiquement le pouvoir de décision sur la formation}

Il s'agit de restituer le pouvoir aux acteurs afin de leur permettre de prendre conscience des enjeux des politiques de la formation continue. Ce n'est pas simple pour une institution éducative qui fonde sa cohérence sur un appareil très hiérarchisé. Les réactions spontanées des acteurs en son sein sont forcément imprégnées de cette culture d'organisation, soit qu'ils répondent de manière très conformiste, soit qu'ils évitent toute expression personnelle (FRIEDBERG E., 1988). C'est pourquoi il est déterminant de mettre en place une décentralisation des systèmes de choix et de prise de décision en matière de formation. Nous pouvons remarquer que cette conduite est en parfaite cohérence avec les choix législatifs qui ont attribué l'autonomie aux établissements d'enseignement. Or, nos observations montrent que très souvent les décisions prises ne vont pas dans ce sens. Elles n'ont pas pour conséquence d'inviter les acteurs à une démarche responsable, mais le contraire. Décentraliser le pouvoir c'est aussi donner aux acteurs de terrain les moyens de connaitre les potentiels disponibles. C'est donc faire œuvre de transparence sur les objectifs et les moyens. Il importe que l'expression de la demande puisse se faire en connaissance des ressources et des contraintes. C'est dans cet espace où l'on est capable d'évaluer les possibles que les projets prennent forme, que les solutions opérationnelles s'élaborent (BOUTINET J.-P., 1990). Par excellence, le lieu d'élaboration de ces choix c'est l'établissement, collectivité cohérente de travail et de projets où une négociation peut naître et prospérer avec ses enjeux, ses stratégies. C'est là qu'apparaissent des propositions, des solutions jusqu'alors impensées car impensables depuis le centre hiérarchique.

\section{Communiquer sur la formation des personnels}

Depuis la création des Missions à la Formation des Personnels de l'Education Nationale à la suite du rapport de PERETTI (1982), au fil des ans, la formation des personnels a pris dans l'Education Nationale une place reconnue, significative. Néanmoins, cette place n'est pas perçue de la même manière par les différents acteurs. En particulier, les enseignants et les personnels administratifs et de service ont une représentation de la formation fort éloignée de la réalité. Ils en méconnaissent largement les structures, les ressources et les limites. Communiquer s'impose donc comme une nécessité si l'on veut faire connaître ce qu'est le dispositif de formation continue des personnels. Une politique de communication bien conduite permet de réduire l'effet des rumeurs qui se développent inévitablement dans ce type d'organisation si l'accès à l'information n'est pas facilité. Elle permet aussi de donner des arguments à ceux (le camp du mouvement) qui veulent utiliser les potentialités existantes pour faire évoluer positivement notre système d'enseignement contre ceux (le camp de l'immobilisme) qui pensent que la lourdeur administrative aura raison de toutes les initiatives. Sur un plan plus symbolique, une politique de communication donne à voir aux agents ce que l'institution souhaite vraiment : la formation continue des personnels est une priorité. C'est pour l'institution dire en actes (de communication) que se former est très important, qu'il faut que tout le monde le sache, qu'il serait aberrant d'être un organisme qui fait métier de la formation et de ne pas se préoccuper au plus haut point de la formation de ses propres agents. Pour atteindre ces buts, la politique de communication cherche à être ouverte, créative. Elle 
choisit une cible large, cherche à atteindre tous les agents. Elle reflète aussi la réalité, témoigne d'une écoute des personnes à qui elle s'adresse.

\section{Evaluer, toujours et encore, ce qui se fait en formation}

La généralisation d'une démarche d'évaluation des actions de formation est un élément décisif pour favoriser une genèse efficace de la demande de formation. On sait que l'existence d'une telle démarche est, par elle-même, un facteur d'amélioration de la qualité. On sait aussi qu'en associant les acteurs à l'évaluation, on contribue à l'évolution positive de l'appareil de formation. C'est en effet en s'appuyant sur le bilan des actions passées que les acteurs peuvent découvrir collectivement et individuellement leurs propres besoins. En ce sens, comme nous l'avions montré à l'occasion d'une expérience menée dans le département du Gard (AUZIOL E. et ETIENNE R., 1987) et plus récemment pour une académie (MAFPEN, 1994), le dispositif d'évaluation peut fournir une véritable structure d'animation pour la formation des personnels. Cela suppose que ce dispositif soit distinct de la structure de formation, qu'il soit décentralisé dans les établissements, qu'une personne reconnue dans l'institution le prenne en charge. Enfin, il ne faut pas oublier que les agents décoderont forcément l'attention portée à l'évaluation comme une affirmation de l'intérêt porté à la formation.

\section{Anticiper l'adaptation de l'appareil administratif aux changements de la formation}

31 Toute transformation dans les procédures concernant la préparation et la mise œuvre de la formation bouscule les habitudes, les façons de faire et risque donc de se heurter à l'inertie de l'appareil. Afin de prévenir cet "effet inertie", il faut veiller à :

1. analyser les difficultés rencontrées pour prendre les décisions qui s'imposent sans remettre en cause les objectifs généraux ; ce qui implique l'existence d'une structure propre à mener cette analyse,

2. tolérer comme inévitables des flottements dans l'organisation durant cette phase d'ajustement. Ces difficultés sont en effet plus souvent le signe d'un changement en cours que le symptôme d'un échec. Il nous faut donc renoncer à l'idée que le changement s'effectuerait d'une manière linéaire et sans à-coups.

32 Le pilotage d'un changement organisationnel nécessite d'autres moyens que ceux que réclame la gestion quotidienne.

\section{CONCLUSION}

Les quelques pistes que nous venons d'esquisser ne sauraient à elles seules garantir une "libération" de la demande de formation; mais une attention persévérante à ces points particuliers permettra de rendre cette demande plus présente et pressante. Nous faisons même l'hypothèse que le mouvement sera amplifié par les interactions entre la formation initiale des personnels et leur formation continue. On peut, en effet, observer ce brassage depuis la mise en place des Instituts Universitaires de Formation des Maîtres (IUFM). La formation des personnels repose sur des projets personnels alimentés à la double source de l'établissement (la réalité des situations professionnelles) et des organismes de formation et de recherche (MAFPEN, IUFM et Universités). Cette alternance provoque un 
retour réflexif sur ce qui fait problème et ce qui apparait comme une voie vers la solution. Nous nous proposons de revenir dans un prochain article sur le rôle de l'établissement dans la formation initiale des personnels.

Un examinateur en Histoire

S. A. : Les enseignants vus au $\mathrm{XIX}^{\circ}$ siècle

(Paris, Editions Errance, 1984, s. p.)

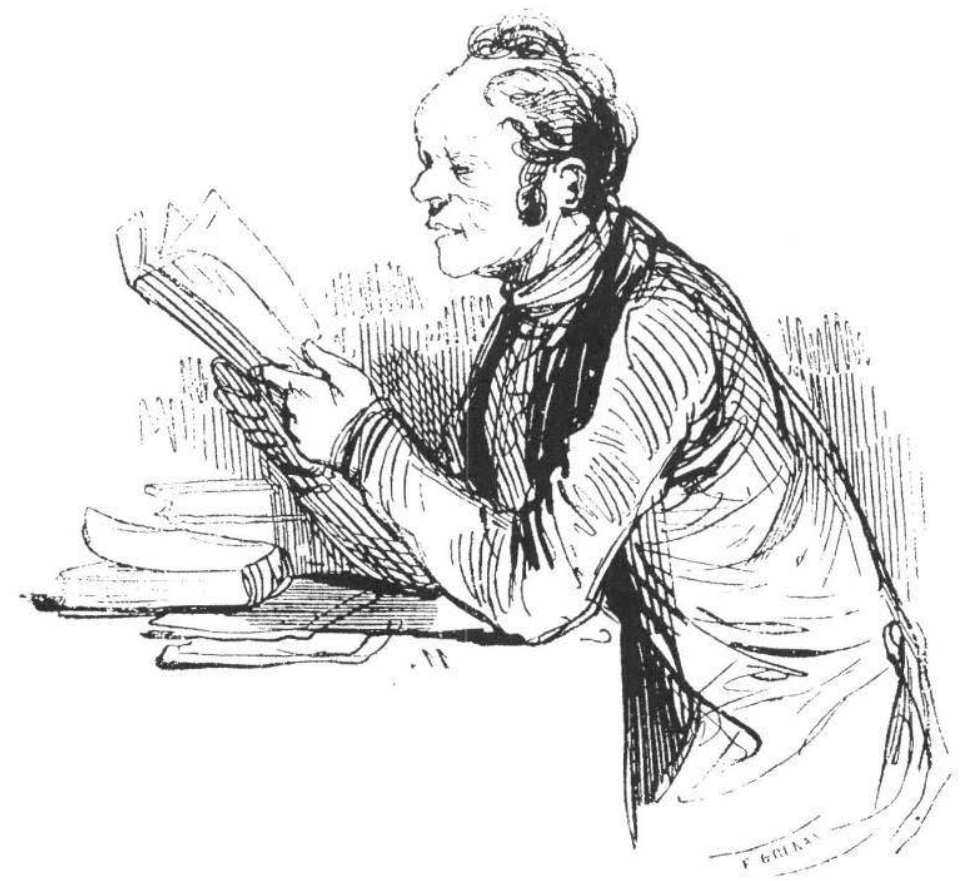

\section{BIBLIOGRAPHIE}

\section{Bibliographie}

AUZIOL E. et NEGRELL M., 1983, Analyse des besoins de formation d'un lycée, MAFPEN, document reprographié, Montpellier.

AUZIOL E. et ETIENNE R., 1987, Commission de formation et évaluation par l'établissement, revue Collèges, numéro 14, MAFPEN, Aix-Marseille.

BOUTINET J.-P., 1990, Anthropologie du projet, PUF, collection Psychologie d'aujourd'hui, Paris.

ETIENNE R., BALDY A. et R., BENEDETTO P., 1992, Le projet personnel de l'élève, Hachette Education, collection Pédagogies pour demain, série Nouvelles Approches, Paris.

ETIENNE R. et AMIEL M., 1994, La communication dans l'établissement scolaire, Hachette Education, collection Pédagogies pour demain, série Nouvelles Approches, Paris. 
FRIEDBERG E., 1988, “L'analyse sociologique des organisation”,POUR, numéro 28.

KAPFERER J.-N., 1987, Rumeurs, le plus vieux média du monde, Le Seuil, Paris.

MAFPEN, Extraits du rapport d'évaluation 92-93, Académie de Montpellier.

MEIGNANT A., 1986, La formation : atout stratégique pour l'entreprise, Les Editions d'Organisation, Paris.

OBIN J.-P., 1993, La crise de l'organisation scolaire, Hachette Education, Paris.

PERETTI A. de, 1982, Rapport au Ministre de l'Education Nationale sur la formation des Personnels de l'Education Nationale, La Documentation Française, Paris.

POSTIC M. et de KETELE J.-M., 1988, Observer les situations éducatives, PUF, collection Pédagogie d'aujourd'hui, Paris.

WATZLAWICK P., WEAKLAND J., FISH R., 1975, Changements, paradoxes et psychothérapie, Le Seuil, collection Points, Paris.

\section{RÉSUMÉS}

Nous étudions ce qui freine le développement de la formation continue des personnels dans les établissements secondaires français et nous établissons les raisons pour lesquelles la stratégie mise en place dans les années 1980 à 1990 n'a pas encore donné son plein effet. Comme cette logique reposant sur la demande individuelle ou collective est la plus efficace, nous proposons de l'accompagner de mesures concrètes et simples qui font de l'établissement l'unité opératoire de la formation.

We study what inhibits the development of the inservice training of secondary school staff in France and we lay clown the reasons why the strategy put in place from the 1980's to the 1990's has not yet reached its full impact. As this logic based on individual or collective demand is the most efficient, we propose with this demand, concrete and simple measures which make the school the operational unit of training.

INDEX

Mots-clés : besoin de formation, demande de formation, offre de formation, plan de formation, projet d'établissement

Keywords : demand of training, need of training, offer of training, program of training, school project

\section{AUTEURS}

\section{ERIC AUZIOL}

Maître de conférences en Sciences de l'information et de la communication à l'université Paul Valéry de Montpellier

\section{RICHARD ETIENNE}

Agrégé de lettres et chargé de mission de formation à l'IUFM de Montpellier 


\section{NADINE MILHAUD}

IPR-IA de mathématiques dans l'académie de Toulouse, ex-directrice adjointe de l'IUFM de Montpellier 